not dream of. We have no right to assume that there may not be condensational vibration in the luminiferous ether. We only do know that any vibrations of this kind which are excited by the reflection and refraction of light are certainly of very small energy compared with the energy of the light from which they proceed. The fact of the case as regards reflection and refraction is this: that, unless the luminiferous ether is absolutely incompressible, the reflection and refraction of light must generally give rise to waves of condensation. Waves of distortion may exist without waves of condensation, but waves of distortion cannot be reflected at the boundary surface between two mediums without exciting in each medium a wave of condensation. When we come to the subject of reflection and refraction we shall see how to deal with these condensational waves, and find how easy it is to get quit of them by supposing the medium incompressible. But it is always to be kept in mind to be examined into: Are there or are there not very small amounts of condensational waves generated in reflection and refraction; and may, after all, the electric force not depend on the waves of condensation? Suppose that we have at any place in air, or in luminiferous ether, a body that, through some action we need not describe, but which is conceivable, is alternately positively and negatively electrified : may it not be that this will be the cause of condensational waves?" It is then supposed that two spherical conductors are connected to the terminals of an alternating dynamo machine, and the quotation proceeds:-

"It is perfectly certain, if we turn the machine slowly, that in the neighbourhood of the conductors we will have alternately positively and negatively electrified elements with reversals perhaps two or three hundred per second of time, without a gradual transition from negative through zero to positive, and the same thing all through space; and we can tell exactly what is the potential at each point. Now, does any one believe that, if that revolution was made fast enough, the electrostatic law would follow? Every one believes that, if that process be conducted fast enough several million times, or millions of million times per second, we should be far from fulfilling the electrostatic law in the electrification of the air in the neighbourhood. It is absolutely certain that such an action as that going on would give rise to electrical waves. Now it does seem probable to me that electrical waves are condensational waves in luminiferous ether, and probably it would be that the propagation of these waves would be enormously faster than the propagation of ordinary light waves. I am quite conscions, when speaking of this, of what has been done in the so-called electro-magnetic theory of light. I know the propagation of electric impulse along an insulated wire surrounded by gutta-percha, which I worked out myself about the year I854, and in which I found a velocity comparable with the velocity of light. . . That is a very different case from this, and I have waited in vain to see how we can get any justification of the way of putting it in the socalled electro-magnetic theory of light."

In those parts of the lectures which deal with wave propagation in an isotropic medium, by far the most interesting parts are those which treat of the conditions at bounding surfaces, whether these surfaces be reflecting and refracting surfaces or surfaces of radiating molecules, or surfaces of absorbing molecules. Lord Ravleigh's investigations and his own on the likelihood of the density or the rigidity of the substance composing a molecule differing from that of the ether are also full of interest

Much of this part of the subject has been thoroughly worked out before, but here the treatment is so original, the language is so suggestive, and I need hardly say that the whole course of lectures is so pregnant with useful ideas, that every one who reads this part will be well repaid.
Having now roughly indicated the novel points and the general mode of treatment of the problem in molar dynamics, I propose in the next notice to give some account of the problem in molecular dynamics, which occupied half of the time. GEORGE FORBES

( $T o$ be continued.)

\section{THE LONG DURATIONS OF METEORIC RADIANT POINTS}

$\mathrm{I} \mathrm{T}$ is unfortunate that the observation of shooting stars is associated with difficulties of no common order. The very large number of distinct showers visible at the same epoch, their extremely attenuated character, and the many impediments to accurate determinations of the flights of the individual shooting stars proceeding from them, exercise an unfavourable influence on the work and deter many observers from grappling with a subject which is admittedly beset with such perplexing details. Apart from this, there exists the great necessity for observations to be sustained during the whole night, and this is rarely practicable either by amateur or professional astronomers, who generally have other important work in hand. In fact, meteoric astronomy requires the almost exclusive attention of the observer, and must be closely pursued for a long period of time if anything like comprehensive results are to be obtained. The voids occasioned either by moonlight or cloudy weather in a short series of observations are only to be filled up by prolonged watches extended over many consecutive years.

The long visible duration of a large number of radiant points of shooting stars is, it must be confessed, a fact which defies satisfactory explanation. The ingenious theory which had attributed to meteor streams an identity with cometary orbits, required that the visibility of such streams should be of very brief character, though in the case of an abnormally wide system or of a shower directed from a point near the earth's apex the duration might be longer than usual, but the radiant point could not maintain a perfectly fixed position amongst the stars. This general view of the subject is, however, not accordant with the results of recent observations, for while there are undoubtedly some cometary showers which display all the peculiarities taught by theory, there are many other streams which continue visible for several months and retain a stationary position in the firmament. It is evident therefore that these streams are presented to us under totally different circumstances as regards orbit to the true planetary showers, and are amenable to conditions and laws which form a problem the solution of which is arrested by no ordinary difficulties.

The multiplicity of streams would naturally originate a false appearance of long duration in certain radiant points, but observations of very precise character would soon show that the point of radiation, as successively determined, differed considerably, being not, in fact, confined absolutely to the same point in the sky. But it is now proved that there are no differences, other than those introduced by small unavoidable errors of observation, in the centres from which shooting stars continue to fall during several months. Indeed, it seems a probable inference from the observations that some showers exist all the year round, though not visible during the epoch when they are very near to the sun.

That such long enduring radiants of meteors can have a community of origin and belong to physically associated streams in the same degrce as the true cometary meteor showers is very difficult to understand. But the fixity of the radiant over so long an interval would yet seem to indicate some bond of close affinity existing between them. At any rate we have no reason to suppose that a large number of showers, distinct in themsclves, can occur consecutively from the same points of the sky owing to a common peculiarity of grouping. 
At intervals of six months the earth's motion in space is in exactly opposite directions, and yet these streams of meteors enter the atmosphere from the same apparent radiants. Evidently therefore the meteoric particles, which individually move in parallel flights, are travelling independent of solar attraction and are presented to us under a totally different aspect to the cometary showers the phenomena of which are clearly understood.

If meteoric streams of great width are encountered by the earth as the result of the sun's proper motion in space then it would appear that to give the phenomena of stationary radiants they must move with enormous velocities. This is not borne out by the observations, for the meteors of these long-enduring streams exhibit appearances similar to what is generally observed in the meteors from the cometary showers. The farther the radiant is removed from the earth's apex the slower become the motions of the meteors, they lose the streak-generating capacity, and their colour changes from white to yellow or red, indicating a lower degree of incandescence as the result of a less violent friction with the atmosphere. There are exceptions, howcver, for the meteors from some radiants retain a velocity much greater than that theoretically assigned.

There is a very pressing need for further observations specially directed to the visible trajectories of shooting stars. The apparent motions of the corpuscles belonging to a stream depend upon several conditions which are very liable to originate discordances. The particles near the radiants move slowly in short courses owing to foreshortening, and when the radiant is near the horizon the flights are longer and more gradual than when it has reached a considerable altitude. The Geminids of December, for instance, appear very slow in the early hours of the evening, but in the morning their swift, diving courses would lead the observer to attribute them to an entirely separate family were it not that the radiant occupies an identical place to that determined

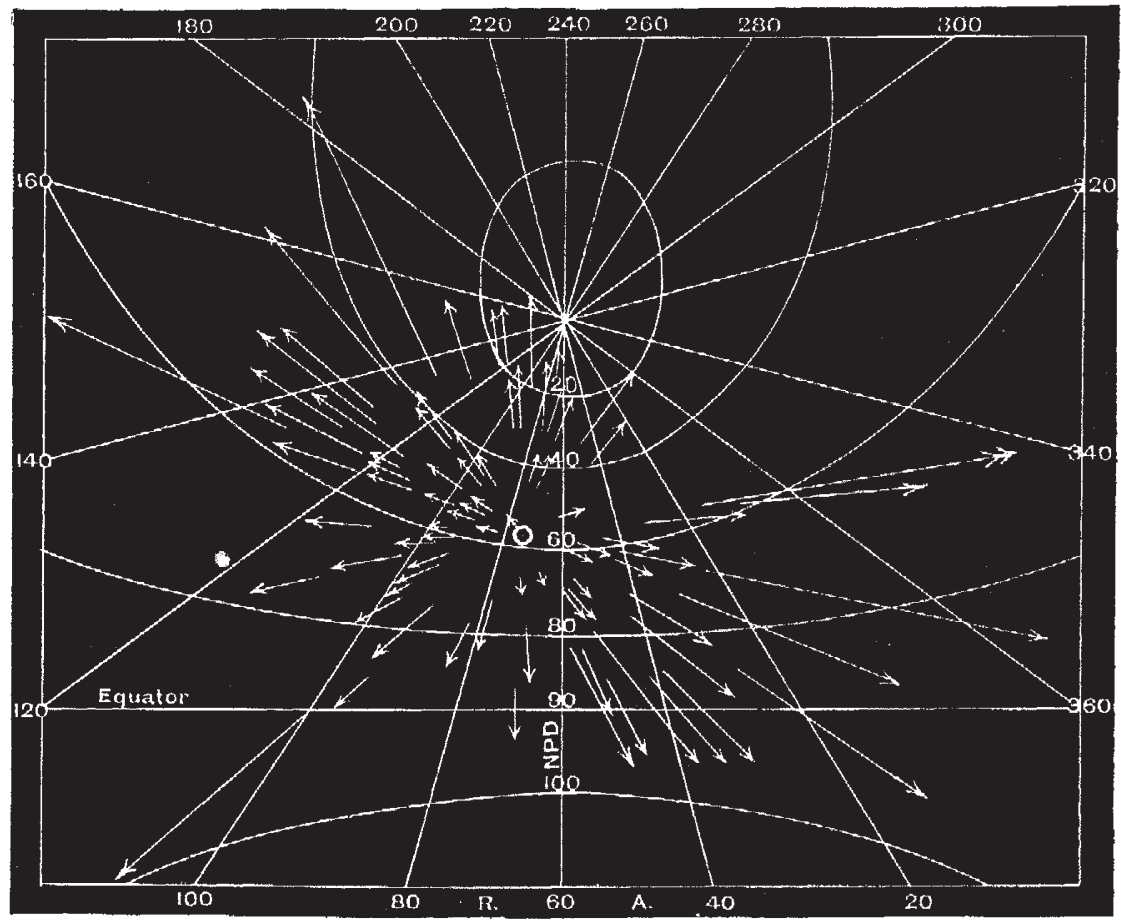

F.,. T.

from the slower meteors recorded several hours before. Some showers also doubtless furnish meteors which become igneous at greater distances than others and more relatively slower than those bclonging to streams formed of materials not so readily combustible. Moreover the specific gravity of the particles of different systems probably varies to some extent, and their individual forms may not always coincide, so that the effects of atmospheric resistance must necessarily introduce peculiarities in the observed flights.

The idea occurred to me that these long-enduring radiants must result from terrestrial meteor streams, i.e. streams revolving around the earth in an excentric orbit with perigee near the outer limits of the atmosphere. If streams of this character cxisted and were closing in upon the earth we should have the phenomena of stationary radiants. And the fact of their closing in upon us would be rendered possible on the assumption of a resisting medium (similar to that affecting the motion of Encke's comet), or that at each return to perigee the atoms en- countered the tenuous outer region of the atmosphere, which, though not sufficiently dense to render them incandescent, would slightly diminish their velocity and thtis bring about a contraction of the orbit. But there are difficulties to the adoption of such views, one of which is that the meteors from such streams would exhibit a consistency of velocity whatever the relative position of their radiants with regard to the earth's direction of motion, and this does not accord with the facts 'of observation.

The earth's atmosphere probably extends in a barely appreciable degree a much greater distance than ordinary estimates allow. The computed heights of certain mcteors deduced from multiple observations, and the phenomena of minute, telescopic shooting stars, which are evidently far exterior to ordinary naked eye meteors, render this highly probable. The former are very numerous, though to what degree is only known to those who have been habitually engaged in sweeping the heavens with a telescope of low power and large field. According to my 
own observations telescopic meteors exceed the more conspicuous class of these bodies in the proportion of about 40 to I. Rich showers probably exist only visible with instrumental means, and certain showers readily perceptible to the naked eye afford little indication of their existence with telescopic aid. The Geminids may be ranked among the latter, for on December 12, 1877, Lewis Swift at Rochester, whilst comet seeking during a period of $4 \frac{1}{2}$ hours, noticed a large number of naked eye meteors. They frequently intruded upon his attention in the intervals when his eye was withdrawn from the telescope, and his estimate of the number visible was 1000 for the whole period of his observations. Yet, singularly enough, there was an unusual paucity of telescopic meteors, only two certainly, and one other suspected, crossing the field of view of $\mathrm{I}_{2}{ }^{\circ}$, whereas they are usually of frequent occurrence.

The observation of meteors, both telescopic and otherwise, especially commends itself to amateurs as an attractive study, requiring no elaborate or expensive instruments. The inconveniences attending such work may soon in great measure be overcome by patience. In cases where the results are thoroughly reliable we think that even slender observations possess weight and ought to be encouraged, for such results soon accumulate, and if

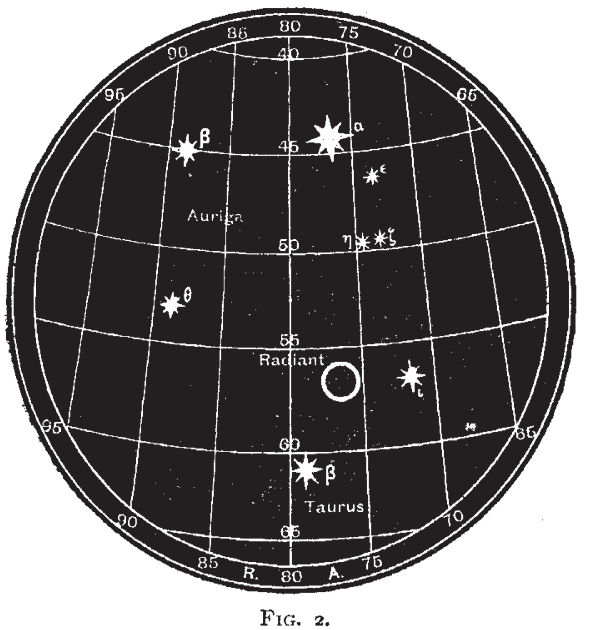

allowed to extend over several years may be combined and reduced to very satisfactory issues. Or the materials obtained by different observers for similar epochs might be collected and the radiant points determined from a careful analysis of the path-directions. In every instance, however, the physical appearances of the meteors ought to be fully described and given due weight in fixing the radiants. Without these precautions it is impossible to obtain reliable positions or to arrange the meteors into family groups with anything like that precision which is an essential feature of the work.

It is earnestly hoped that more enthusiasm may now be aroused amongst observers in this interesting department of astronomy. The question as to the duration of radiants and their absolutely fixed position must have an important bearing on the theory of their origin, and deserves much further investigation. The apparently intermittent character of many such streams also deserves notice, as their fluctuations may be regulated by definite periods of short duration. The observations of individual radiants should be confined to a few nights only, or when, from the paucity of meteors, it is found necessary to include results extending over several weeks, the nights of greatest intensity should be mentioned. The necessity exists for cqual accuracy in deducing the radiant points, as in registering the exact directions of flight; in fact, great discrimination and precision are required in details so mutually dependent, so liable to errors, and so full of complications.

One of the most active and at the same time one of the most precise and well-defined cases of long duration is exhibited by a meteor shower in the southern extremity of Auriga and slightly to the north-east of a line connecting the stars $\iota$ Aurigæ and $\beta$ Tauri. It gives the first sign of its existence at the end of July, and thence continues during several ensuing months. The epochs about October 8-I 5 and November 7 and 20 would appear to represent the most prominent exhibition of this radiant, though there are many other nights during the summer and autumnal months when it may be detected during a prolonged watch. The accompanying diagram (Fig. I) shows the projected paths of eighty meteors (chiefly observed by myself at Bristol, and selected as being tolerably near the radiant point) recorded during the months of October and November. These paths form only a proportion of the aggregate number seen, but they sufficiently display the singularly precise radiation of this stream. A similar diagram might readily be prepared from the flights recorded in August and September when the convergence of an almost equal number of meteors attest the visibility of the same radiant. Its position relatively to the stars is given in Fig. 2, and it is hoped that observers will endeavour to effect its re-observation. We require further observations particularly during the month of August, when the radiant is very low, until the morning hours, and this doubtless accounts for the rareness of its apparition at that epoch.

W. F. DENNING

\section{NOTES}

THOSE who are interested in the South Kensington Museum will be glad to learn that the National Collections belonging to the Science Department have now a prospect of improvement. A Government Committee has lately been appointed to report generally upon them and to consider plans for properly housing them. The Committee consists of gentlemen of high position in various Government Departments ; the Chairman is Sir Frederick Bramwell, F.R.S., and Dr. W. Pole, F.R.S., is the Secretary.

The Prince of Wales, President of the International Inventions Exhibition, has fixed Monday, May 4, for the opening of the Exhibition. Rapid progress is being made in all branches connected with the Exhibition. The large space set apart for machinery in motion is already being filled, whilst preparations for receiving other exhibits are well forward, some of which have arrived at the building. The Aquarium Department is receiving considerable attention, and will form a very attractive feature. The tanks have been thoroughly cleansed and refilled with fresh water, which has been softened and filtered, rendering it bright and pure, fit for the reception of large consignments of fish that will shortly arrive. Lord Onslow has lately presented I500 exceedingly fine carp to the Aquarium, and a large number of fish indigenous to the Canadian Lakes have also been received for exhibition in the tanks.

THE Royal Society of New South Wales offers its medal and a money prize for the best communication (provided it be of sufficient merit) containing the results of original research or observation upon each of the following subjects:-Series IV. To be sent in not later than May I, I885:-No. I3. Anatomy and life-history of the Echidna and Platypus; the Society's Medal and 25l. 14. Anatomy and life-history of Mollusca peculiar to Australia; the Society's Medal and 25l. 15. The chemical composition of the profucts from the so-called Kerosene Shale of New South Wales; the Society's Medal and $25^{l}$. Series V. To be sent in not later than May I, I886:-No. 16. On the chemistry of the Australian gums and resins; the 